\title{
Shape Operator via Darboux Frame Curvatures and Its Applications
}

\author{
Bahar Uyar Düldüi* and Mustafa Düldiul \\ (Communicated by Kazım İlarslan)
}

\begin{abstract}
We present the shape operator's matrix of a surface along a surface curve. By using the obtained matrix, we give a short proof of the Beltrami-Enneper theorem. Also, we give a new method for determining the well-known geodesic curves of a plane, a sphere and a circular cylinder by using the relation between the ordinary curvatures of the geodesic and the curvatures of the surface.
\end{abstract}

Keywords: Shape operator; Darboux frame; geodesic curve; curvature.

AMS Subject Classification (2010): Primary: 53A04; Secondary: 53A05.

\section{Introduction}

In Euclidean 3-space, the shape of a regular curve is measured by its curvature functions, called curvature and torsion. If the curve lies on a regular surface, it has also curvatures with respect to the surface itself, called normal curvature, geodesic curvature and geodesic torsion. These functions determine important properties for surface curves. When these curvatures vanish along the curve, such curves are called as asymptotic curve, geodesic curve, and line of curvature, respectively. Similar to curves, we can measure the shape of a surface. To do this, we need to determine the shape operator of the surface. We can compute the shape operator of a surface by using its definition or the Weingarten equations. Using Weingarten equations yield the shape operator depending on the first and second fundamental form coefficients of the surface.

The purpose of this paper is to give a new method for determining the shape operator of a surface. Our new method is based on the Darboux frame of a surface curve. By using the Darboux frame curvatures of the curve, we obtain the shape operator of a surface along the curve depending on the normal curvature and geodesic torsion of the surface curve. We then give the mean, Gaussian and principal curvatures depending on these curvatures. As an application, we give a short proof of the Beltrami-Enneper theorem, and we obtain the relation between the ordinary curvatures of the surface curve and the curvatures of the surface. Also, we give a new and easy method for determining the well-known geodesic curves of a plane, sphere and circular cylinder.

\section{Preliminaries}

Let us consider a unit speed curve $\beta$ lying on a regular surface $\mathcal{M}$ in Euclidean 3-space $\mathbb{E}^{3}$. Along $\beta$, we denote the unit tangent vector of the curve with $\mathcal{T}$, the unit normal vector of the surface with $\mathcal{U}$, and $\mathcal{V}=\mathcal{U} \times \mathcal{T}$. In this case, we call the frame $\{\mathcal{T}, \mathcal{V}, \mathcal{U}\}$ as Darboux frame. This frame has its own Frenet formulas given by

$$
\left\{\begin{array}{l}
\mathcal{T}^{\prime}=\kappa_{g} \mathcal{V}+\kappa_{n} \mathcal{U} \\
\mathcal{V}^{\prime}=-\kappa_{g} \mathcal{T}+\tau_{g} \mathcal{U} \\
\mathcal{U}^{\prime}=-\kappa_{n} \mathcal{T}-\tau_{g} \mathcal{V}
\end{array},\right.
$$

where $\kappa_{g}$ is the geodesic curvature, $\kappa_{n}$ is the normal curvature and $\tau_{g}$ is the geodesic torsion of $\beta$ [2]. 
Definition 2.1. Let $\mathcal{M}$ be a regular surface oriented with the unit normal $U$. The shape operator $S: T_{P}(\mathcal{M}) \rightarrow$ $T_{P}(\mathcal{M})$ of $\mathcal{M}$ at the point $P \in \mathcal{M}$ is defined by $S\left(\mathrm{X}_{P}\right)=-\mathcal{D}_{\mathrm{X}_{P}}^{U}$ for $\mathrm{X}_{P} \in T_{P}(\mathcal{M})$, where $T_{P}(\mathcal{M})$ denotes the tangent plane of the surface at $P$.

Definition 2.2. Let $\mathcal{M}$ be a regular surface in $\mathbb{E}^{3}, P \in \mathcal{M}$ be a point and $S_{P}$ denotes the shape operator of $\mathcal{M}$ at $P$. The Gaussian and mean curvatures of $\mathcal{M}$ at $P$ are defined by $\mathcal{K}(P)=\operatorname{det} S_{P}$ and $\mathcal{H}(P)=\frac{1}{2} \operatorname{trace}\left(S_{P}\right)$, respectively. Also, the eigenvalues of $S_{P}$ are called the principal curvatures of $\mathcal{M}$ at $P$.

Definition 2.3. Let $\mathcal{M}$ be a regular surface in $\mathbb{E}^{3}, P \in \mathcal{M}$ be a point, and $\mathrm{X}_{P} \in T_{P}(\mathcal{M})$ be a unit tangent vector. Then $\kappa_{n}\left(\mathrm{X}_{P}\right)=\left\langle S\left(\mathrm{X}_{P}\right), \mathrm{X}_{P}\right\rangle$ is called the normal curvature at $P$ of $\mathcal{M}$ in the direction of $\mathrm{X}_{P}$. If $\kappa_{n}\left(\mathrm{X}_{P}\right)$ is constant for all $\mathrm{X}_{P} \in T_{P}(\mathcal{M})$, then $P$ is called umbilic point on $\mathcal{M}$. If $\kappa_{n}\left(\beta^{\prime}\right)=0$ along a regular curve $\beta$ lying on $\mathcal{M}$, then $\beta$ is called an asymptotic curve on $\mathcal{M}$.

Lemma 2.1. Let $\mathcal{M}$ be a regular surface parametrized by $X(u, v)$ and $\beta(s)=X(u(s), v(s))$ be a unit speed curve lying on $\mathcal{M}$. Then, the normal curvature of the curve is obtained by [4]

$$
\kappa_{n}=\mathrm{L}\left(u^{\prime}\right)^{2}+2 \mathrm{M} u^{\prime} v^{\prime}+\mathrm{N}\left(v^{\prime}\right)^{2},
$$

and the geodesic torsion of the curve is obtained by [3]

$$
\tau_{g}=\frac{1}{\sqrt{\mathrm{EG}-\mathrm{F}^{2}}}\left\{(\mathrm{EM}-\mathrm{FL})\left(u^{\prime}\right)^{2}+(\mathrm{EN}-\mathrm{GL}) u^{\prime} v^{\prime}+(\mathrm{FN}-\mathrm{GM})\left(v^{\prime}\right)^{2}\right\},
$$

where $\mathrm{E}, \mathrm{F}, \mathrm{G}$ and $\mathrm{L}, \mathrm{M}, \mathrm{N}$ denote the first and second fundamental form coefficients of the surface, respectively.

Lemma 2.2. Let $\mathcal{M}$ be a regular surface given by $f(x, y, z)=0$ and $\beta(s)=(x(s), y(s), z(s))$ be a unit speed curve lying on $\mathcal{M}$. Then, the normal curvature of the curve is obtained by [4]

$$
\kappa_{n}=-\frac{f_{x x}\left(x^{\prime}\right)^{2}+f_{y y}\left(y^{\prime}\right)^{2}+f_{z z}\left(z^{\prime}\right)^{2}+2\left(f_{x y} x^{\prime} y^{\prime}+f_{y z} y^{\prime} z^{\prime}+f_{x z} x^{\prime} z^{\prime}\right.}{\sqrt{f_{x}^{2}+f_{y}^{2}+f_{z}^{2}}}
$$

and the geodesic torsion of the curve is obtained by [3]

$$
\tau_{g}=\frac{1}{\|\nabla f\|}\left\{\left(a_{3} f_{y}-a_{2} f_{z}\right) x^{\prime}+\left(a_{1} f_{z}-a_{3} f_{x}\right) y^{\prime}+\left(a_{2} f_{x}-a_{1} f_{y}\right) z^{\prime}\right\},
$$

where

$$
\begin{aligned}
& a_{i}=\frac{1}{\|\nabla f\|}\left(f_{x_{i} x_{i}} x_{i}^{\prime}+f_{x_{i} x_{j}} x_{j}^{\prime}+f_{x_{i} x_{k}} x_{k}^{\prime}\right)-\frac{1}{\|\nabla f\|^{3}}\left\{f_{x_{i}}^{2}\left(f_{x_{i} x_{i}} x_{i}^{\prime}+f_{x_{i} x_{j}} x_{j}^{\prime}+f_{x_{i} x_{k}} x_{k}^{\prime}\right)\right. \\
& \left.+f_{x_{i}} f_{x_{j}}\left(f_{x_{j} x_{i}} x_{i}^{\prime}+f_{x_{j} x_{j}} x_{j}^{\prime}+f_{x_{j} x_{k}} x_{k}^{\prime}\right)+f_{x_{i}} f_{x_{k}}\left(f_{x_{k} x_{i}} x_{i}^{\prime}+f_{x_{k} x_{j}} x_{j}^{\prime}+f_{x_{k} x_{k}} x_{k}^{\prime}\right)\right\}
\end{aligned}
$$

with $x_{1}=x, x_{2}=y, x_{3}=z(i, j, k=1,2,3$ cyclic $)$.

\section{Shape operator's matrix along a surface curve}

In this section, we consider a regular curve lying on an oriented surface in Euclidean 3-space and compute the shape operator's matrix of the surface along the curve.

The following two Lemmas will be needed in Proposition 3.1.

Lemma 3.1. Let $\mathcal{M}$ be a regular surface parametrized by $X(u, v) ; \beta(s)=X(u(s), v(s))$ be a unit speed curve lying on $\mathcal{M}$, and $\{\mathcal{T}, \mathcal{V}, \mathcal{U}\}$ denotes the Darboux frame field of $\beta$. Then, the normal curvature of $\mathcal{M}$ in the direction of $\mathcal{V}$ can be obtained by

where

$$
\kappa_{n}(\mathcal{V})=\frac{1}{\mathrm{EG}-\mathrm{F}^{2}}\left(\lambda_{1}\left(u^{\prime}\right)^{2}+2 \lambda_{2} u^{\prime} v^{\prime}+\lambda_{3}\left(v^{\prime}\right)^{2}\right)
$$

$$
\begin{aligned}
& \lambda_{1}=\mathrm{F}(\mathrm{FL}-\mathrm{EM})+\mathrm{E}(\mathrm{EN}-\mathrm{FM}), \\
& \lambda_{2}=\mathrm{F}(\mathrm{GL}-\mathrm{FM})+\mathrm{E}(\mathrm{FN}-\mathrm{GM}), \\
& \lambda_{3}=\mathrm{G}(\mathrm{GL}-\mathrm{FM})+\mathrm{F}(\mathrm{FN}-\mathrm{GM})
\end{aligned}
$$

are computed along $\beta$. 
Proof. Since $\mathcal{T}=\beta^{\prime}(s)=\mathbf{X}_{u} u^{\prime}+\mathbf{X}_{v} v^{\prime}$ and $\mathcal{U}=\frac{\mathbf{X}_{u} \times \mathbf{X}_{v}}{\left\|\mathbf{X}_{u} \times \mathbf{X}_{v}\right\|}=\frac{\mathbf{X}_{u} \times \mathbf{X}_{v}}{\sqrt{\mathrm{EG}-\mathrm{F}^{2}}}$, we have

$$
\begin{gathered}
\mathcal{V}=\mathcal{U} \times \mathcal{T}=\frac{\mathbf{X}_{u} \times \mathbf{X}_{v}}{\sqrt{\mathrm{EG}-\mathrm{F}^{2}}} \times\left(\mathbf{X}_{u} u^{\prime}+\mathbf{X}_{v} v^{\prime}\right) \\
=\frac{1}{\sqrt{\mathrm{EG}-\mathrm{F}^{2}}}\left(-\mathrm{F} u^{\prime}-\mathrm{G} v^{\prime}\right) \mathbf{X}_{u}+\left(\mathrm{E} u^{\prime}+\mathrm{F} v^{\prime}\right) \mathbf{X}_{v} .
\end{gathered}
$$

Thus, we obtain the desired result by using $\kappa_{n}(\mathcal{V})=\langle S(\mathcal{V}), \mathcal{V}\rangle$.

Lemma 3.2. Let $\mathcal{M}$ be a regular surface given by its implicit equation $f\left(x_{1}, x_{2}, x_{3}\right)=0 ; \beta(s)=\left(\beta_{1}(s), \beta_{2}(s), \beta_{3}(s)\right)$ be a unit speed curve lying on $\mathcal{M}$, and $\{\mathcal{T}, \mathcal{V}, \mathcal{U}\}$ denotes the Darboux frame field of $\beta$. Then, the normal curvature of $\mathcal{M}$ in the direction of $\mathcal{V}$ can be obtained by

$$
\begin{array}{r}
\kappa_{n}(\mathcal{V})=\frac{-1}{\sqrt{\left(f_{1}^{2}+f_{2}^{2}+f_{3}^{2}\right)^{3}}} \sum_{i=1}^{3}\left\{\left(f_{j j} f_{k}^{2}+f_{k k} f_{j}^{2}-2 f_{j k} f_{j} f_{k}\right)\left(\beta_{i}^{\prime}\right)^{2}\right. \\
\left.+2\left(f_{j k} f_{i} f_{k}+f_{i k} f_{k} f_{j}-f_{i} f_{j} f_{k k}-f_{i j} f_{k}^{2}\right) \beta_{i}^{\prime} \beta_{j}^{\prime}\right\},
\end{array}
$$

where $i, j, k=1,2,3$ (cyclic) and

$$
f_{r}=\frac{\partial f}{\partial x_{r}}, \quad f_{r s}=\frac{\partial^{2} f}{\partial x_{s} \partial x_{r}}, \quad r, s=1,2,3
$$

are computed along $\beta$.

Proof. Since $\mathcal{T}=\beta^{\prime}(s)=\left(\beta_{1}^{\prime}(s), \beta_{2}^{\prime}(s), \beta_{3}^{\prime}(s)\right)$ and $\mathcal{U}=\frac{\nabla f}{\|\nabla f\|}=\frac{1}{\|\nabla f\|}\left(f_{1}, f_{2}, f_{3}\right)$, we have

$$
\mathcal{V}=\mathcal{U} \times \mathcal{T}=\frac{1}{\sqrt{f_{1}^{2}+f_{2}^{2}+f_{3}^{2}}}\left(f_{2} \beta_{3}^{\prime}-f_{3} \beta_{2}^{\prime}, f_{3} \beta_{1}^{\prime}-f_{1} \beta_{3}^{\prime}, f_{1} \beta_{2}^{\prime}-f_{2} \beta_{1}^{\prime}\right) .
$$

The desired result follows from (2.3).

Proposition 3.1. Let $\mathcal{M}$ be a regular surface, $\beta$ be a unit speed curve lying on $\mathcal{M}$, and $\{\mathcal{T}, \mathcal{V}, \mathcal{U}\}$ denotes the Darboux frame field of $\beta$. Then, the shape operator's matrix of $\mathcal{M}$ along $\beta$ with respect to the basis $\{\mathcal{T}, \mathcal{V}\}$ is given by

$$
S=\left[\begin{array}{cc}
\kappa_{n}(\mathcal{T}) & \tau_{g} \\
\tau_{g} & \kappa_{n}(\mathcal{V})
\end{array}\right]
$$

Proof. We may write

$$
S(\mathcal{T})=-\nabla_{\mathcal{T}}^{U}=-\mathcal{U}^{\prime}=\kappa_{n}(\mathcal{T}) \mathcal{T}+\tau_{g} \mathcal{V}
$$

Let

$$
S(\mathcal{V})=a \mathcal{T}+b \mathcal{V}
$$

Then, we obtain

$$
\begin{gathered}
a=\langle S(\mathcal{V}), \mathcal{T}\rangle=\langle\mathcal{V}, S(\mathcal{T})\rangle=\left\langle\mathcal{V},-\mathcal{U}^{\prime}\right\rangle=\tau_{g}, \\
b=\langle S(\mathcal{V}), \mathcal{V}\rangle=\kappa_{n}(\mathcal{V}),
\end{gathered}
$$

i.e.

$$
S(\mathcal{V})=\tau_{g} \mathcal{T}+\kappa_{n}(\mathcal{V}) \mathcal{V}
$$

Thus, the assertion is clear from (3.5) and (3.6). 


\section{Applications of Main Result}

If we use Proposition 3.1, the following results can be given:

Corollary 4.1. Let $\beta$ be a unit speed curve lying on an oriented surface $\mathcal{M}$, and $\{\mathcal{T}, \mathcal{V}, \mathcal{U}\}$ denotes the Darboux frame field of $\beta$. Then, the curvatures of the surface along $\beta$ depending on the Darboux frame curvatures are given by

$$
\begin{gathered}
\text { Gaussian curvature: } \quad \mathcal{K}=\kappa_{n}(\mathcal{T}) \kappa_{n}(\mathcal{V})-\tau_{g}^{2} \\
\text { Mean curvature: } \quad \mathcal{H}=\frac{1}{2}\left(\kappa_{n}(\mathcal{T})+\kappa_{n}(\mathcal{V})\right) \\
\text { Principal curvatures : } \quad k_{1,2}=\frac{1}{2}\left(\kappa_{n}(\mathcal{T})+\kappa_{n}(\mathcal{V}) \pm \sqrt{\left(\kappa_{n}(\mathcal{T})-\kappa_{n}(\mathcal{V})\right)^{2}+4 \tau_{g}^{2}}\right)
\end{gathered}
$$

Corollary 4.2. If the curve $\beta$ is a line of curvature on $\mathcal{M}$ with non-umbilical points, then $\mathcal{T}$ corresponds to the principal direction, i.e. $\kappa_{n}(\mathcal{T})=k_{1}, \kappa_{n}(\mathcal{V})=k_{2}$. Thus, substituting these results together with $\tau_{g}=0$ into (4.1) and (4.2) we obtain the classical results: $\mathcal{K}=k_{1} k_{2}$ and $\mathcal{H}=\frac{k_{1}+k_{2}}{2}$.

Corollary 4.3. Let $\mathcal{M}$ be a regular surface parametrized by $X(u, v)$, and $\beta(s)=X(u(s), v(s))$ be a unit speed curve lying on $\mathcal{M}$. Then, the Gaussian curvature of the surface along $\beta$ is given by

$$
\begin{aligned}
\mathcal{K}=\frac{1}{\mathrm{EG}-\mathrm{F}^{2}}\{ & \left(\mathrm{L}\left(u^{\prime}\right)^{2}+2 \mathrm{M} u^{\prime} v^{\prime}+\mathrm{N}\left(v^{\prime}\right)^{2}\right)\left(\lambda_{1}\left(u^{\prime}\right)^{2}+2 \lambda_{2} u^{\prime} v^{\prime}+\lambda_{3}\left(v^{\prime}\right)^{2}\right) \\
- & {\left.\left[(\mathrm{EM}-\mathrm{FL})\left(u^{\prime}\right)^{2}+(\mathrm{EN}-\mathrm{GL}) u^{\prime} v^{\prime}+(\mathrm{FN}-\mathrm{GM})\left(v^{\prime}\right)^{2}\right]^{2}\right\}, }
\end{aligned}
$$

and the mean curvature of the surface along $\beta$ is given by

$$
2 \mathcal{H}=\left(\mathrm{L}+\frac{\lambda_{1}}{\mathrm{EG}-\mathrm{F}^{2}}\right)\left(u^{\prime}\right)^{2}+2\left(\mathrm{M}+\frac{\lambda_{2}}{\mathrm{EG}-\mathrm{F}^{2}}\right) u^{\prime} v^{\prime}+\left(\mathrm{N}+\frac{\lambda_{3}}{\mathrm{EG}-\mathrm{F}^{2}}\right)\left(v^{\prime}\right)^{2},
$$

where $\lambda_{i}$ are as given in Lemma 3.1.

Proposition 4.1. Let $\beta$ be a geodesic curve on a regular surface $\mathcal{M} \subset \mathbb{E}^{3}$. Then the relation between the ordinary curvatures of $\beta$ and the curvatures of $\mathcal{M}$ is given by

$$
2 \kappa \mathcal{H}-\mathcal{K}=\kappa^{2}+\tau^{2}
$$

Proof. Since $\beta$ is geodesic on $\mathcal{M}$, we have $\kappa_{g}=0, \kappa_{n}(\mathcal{T})=\kappa$ and $\tau_{g}=\tau$. Then, from (4.1) and (4.2) we obtain

$$
\mathcal{K}=\kappa . \kappa_{n}(\mathcal{V})-\tau^{2}, \quad 2 \mathcal{H}=\kappa+\kappa_{n}(\mathcal{V}) .
$$

If we eliminate $\kappa_{n}(\mathcal{V})$ from these equations, we find (4.4).

If we use our new results, we may also give a short proof of the Beltrami-Enneper theorem:

Theorem 4.1 (Beltrami-Enneper). [1] Let $\beta$ be an asymptotic curve on a regular surface $\mathcal{M} \subset \mathbb{E}^{3}$, and assume the curvature $\kappa$ of $\beta$ does not vanish. Then the torsion $\tau$ of $\beta$ and the Gaussian curvature $\mathcal{K}$ of $\mathcal{M}$ are related along $\beta$ by $\mathcal{K}=-\tau^{2}$.

Proof. Since $\beta$ is asymptotic on $\mathcal{M}$, we have $\kappa_{n}(\mathcal{T})=0$ and $\tau_{g}=\tau$. The assertion is clear from (4.1).

Similar to the Beltrami-Enneper theorem, we may state the following theorem:

Theorem 4.2. Let $\beta$ be a geodesic curve on a minimal surface $\mathcal{M} \subset \mathbb{E}^{3}$. Then the Gaussian curvature $\mathcal{K}$ of $\mathcal{M}$ and the ordinary curvatures of $\beta$ are related along $\beta$ by $\mathcal{K}=-\left(\kappa^{2}+\tau^{2}\right)$.

Proof. The proof follows from (4.4). 


\section{The geodesics of plane, sphere, circular cylinder}

The geodesics of plane, cylinder and sphere are well-known. If we use the relation (4.4), we may give a different way of obtaining the geodesic curves of plane, sphere and circular cylinder.

\subsection{Geodesics of a plane}

Let $\beta$ be a geodesic curve on a plane. Then, since we have $\mathcal{K}=\mathcal{H}=0$ for the plane, the curvatures of a geodesic on planes satisfy $\kappa^{2}+\tau^{2}=0$, i.e. $\kappa=\tau=0$. This means the geodesics of planes are only straight lines.

\subsection{Geodesics of a sphere}

Let $\beta$ be a geodesic curve on a sphere with radius $r$. Then, since we have $\mathcal{K}=\frac{1}{r^{2}}, \mathcal{H}=\frac{1}{r}$ for the sphere, the curvatures of a geodesic on sphere satisfy

$$
\left(\kappa-\frac{1}{r}\right)^{2}+\tau^{2}=0
$$

i.e. $\kappa=\frac{1}{r}, \tau=0$. Then geodesics of spheres are planar curves with constant curvature, i.e. the circles having the same radius with the sphere. This means the geodesics of the spheres are their great circles.

\subsection{Geodesics of a circular cylinder}

Let $\beta$ be a geodesic curve on a circular cylinder with radius $r$. Since we have $\mathcal{K}=0, \mathcal{H}=\frac{1}{2 r}$ for the circular cylinder, then the curvatures of a geodesic on circular cylinder satisfy

$$
\left(\kappa-\frac{1}{2 r}\right)^{2}+\tau^{2}=\frac{1}{4 r^{2}}
$$

i.e.

$$
\kappa=\frac{1}{2 r}+\frac{1}{2 r} \cos \varphi, \quad \tau=\frac{1}{2 r} \sin \varphi
$$

for some $\varphi$. Thus, if $\varphi=2 n \pi, n \in \mathbb{Z}$, we obtain $\kappa=\frac{1}{r}, \tau=0$ which means $\beta$ is a circle with the same radius of cylinder. This means the normal section of a circular cylinder is a geodesic.

If $\varphi=(2 n+1) \pi, n \in \mathbb{Z}$, we obtain $\kappa=\tau=0$ which means $\beta$ is a straight line on the cylinder. This means the rulings of the circular cylinder are also geodesic.

If $\varphi \neq k \pi, k \in \mathbb{Z}$ and $\varphi$ is constant, then $\beta$ is a circular helix on cylinder.

\section{References}

[1] Gray, A., Abbena, E. and Salamon, S., Modern differential geometry of curves and surfaces with Mathematica, 3rd Edition, Chapman Hall CRC, 2006.

[2] O’Neill, B., Elementary Differential Geometry, Academic Press, 1966.

[3] Uyar Düldül, B. and Çalışkan, M., On the geodesic torsion of a tangential intersection curve of two surfaces in $\mathbb{R}^{3}$. Acta Math. Univ. Comenian. 82 (2013), 177-189.

[4] Ye, X. and Maekawa, T., Differential geometry of intersection curves of two surfaces. Comput. Aided Geom. Design 16 (1999), 767-788.

\section{Affiliations}

\section{BAHAR UYAR DÜLDÜL}

ADDRESS: Yildiz Technical University, Dept. of Mathematics and Science Education, 34210, Istanbul-Turkey.

E-MAIL: buduldul@yildiz.edu.tr

ORCID ID: 0000-0003-3281-8918 
MustafA DÜldüL

ADDRESS: Yildiz Technical University, Dept. of Mathematics, 34210, Istanbul-Turkey.

E-MAIL: mduldul@yildiz.edu.tr

ORCID ID: 0000-0002-7306-6006 